Physics in Collision - Zeuthen, Germany, June 26-28, 2003

\title{
SOLAR MODELS AND SOLAR NEUTRINOS
}

\author{
John N. Bahcall \\ School of Natural Sciences, Institute for Advanced Study, \\ Princeton, NJ 08540, USA
}

\begin{abstract}
I summarize 40 years of development of the standard solar model that is used to predict solar neutrino fluxes and then describe the current uncertainties in the predictions. I will also attempt to explain why it took so long, about three and a half decades, to reach a consensus view that new physics is being learned from solar neutrino experiments.
\end{abstract}




\section{Introduction}

I begin in Section 2, with a tribute to Ray Davis and Bruno Pontecorvo. In Section 3, I present a concise history of the development of the standard solar model that is used today to predict solar neutrino fluxes. I describe in Section 4 the currentlyestimated uncertainties in the solar neutrino predictions ${ }^{1}$, a critical issue for existing and future solar neutrino experiments. I also present a formula that gives the ratio of the rates of the ${ }^{3} \mathrm{He}^{3} \mathrm{He}$ and the ${ }^{3} \mathrm{He}-{ }^{4} \mathrm{He}$ reactions as a function of the $\mathrm{p}-\mathrm{p}$ and ${ }^{7}$ Be neutrino fluxes. These reactions are the principal terminating fusion reactions of the p-p chain. In Section 5,I give my explanation of why it took so long for physicists to reach a consensus that new particle physics was being learned from solar neutrino experiments.

\section{Ray Davis and Bruno Pontecorvo}

Before I begin the discussion of the standard solar model, I want to begin by paying tribute to two of the great scientists and pioneers of neutrino astrophysics, Ray Davis (Figure 1) and Bruno Pontecorvo (Figure 2). Bruno first suggested using chlorine as a detector of neutrinos in a Chalk River report written in 1946. Ray followed up on Bruno's suggestion and the careful unpublished feasibility study of Louie Alvarez. Using with care and skill a chlorine detector and reactor neutrinos, Ray showed in 1955-1958 that $\nu_{e}$ and $\bar{\nu}_{e}$ were different. About a decade later, Ray first detected solar neutrinos, laying the foundation for the studies that are so widely discussed today. Bruno recognized that solar neutrinos could potentially tell us something about particle physicists and he laid the foundation for the modern theory of neutrino oscillations.

The solar neutrino saga has been a community effort in which thousands of chemists, physicists, astronomers, and engineers have contributed in crucial ways to refining the nuclear physics, the astrophysics, and the detectors so that the subject could become a precision test of stellar evolution and, ultimately, of weak interaction theory. But, these two wonderful scientists and wonderful human beings started us on a marvelous road of discovery.

Ray's role in the subject, like Bruno's, has been unique. Any historical summary, even of solar models, would be grossly incomplete if it did not emphasize the inspiration provided by Ray's experimental vision. Although Ray never was

\footnotetext{
${ }^{1}$ Where contemporary numbers are required in this review, I use the results from the BP00 solar model, ApJ 555 (2001) 990, astro-ph/0010346.
} 


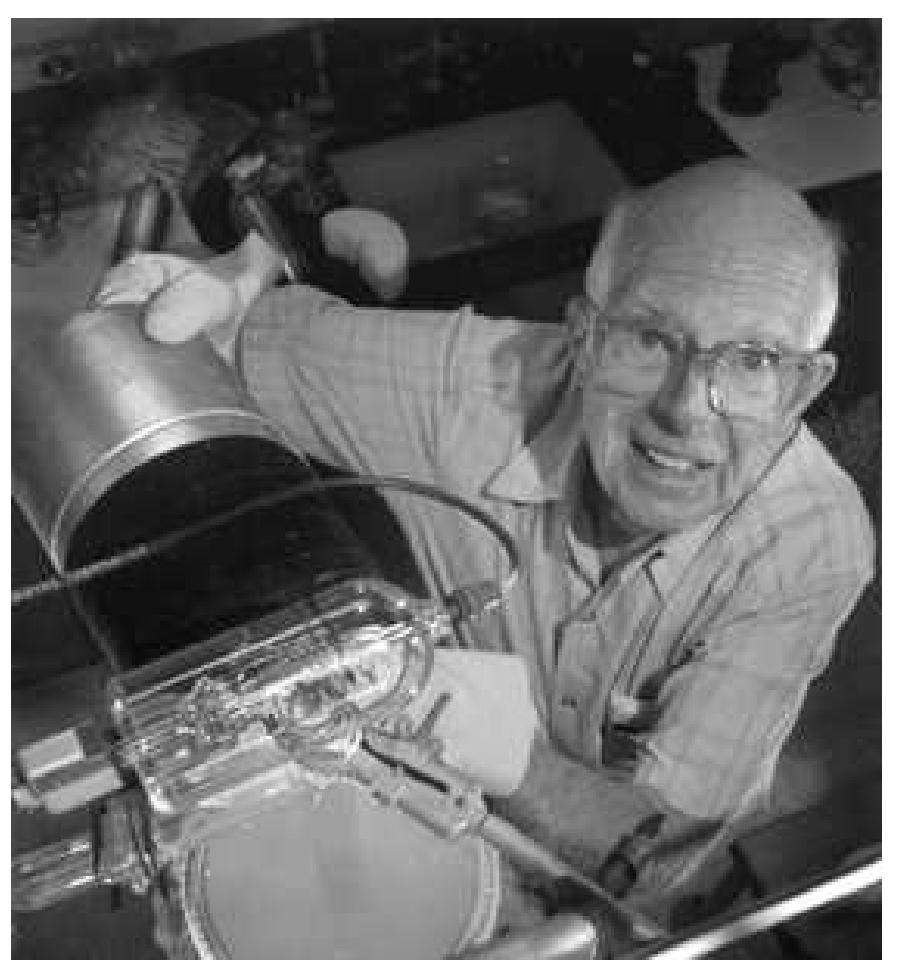

Figure 1: Ray Davis preparing to pour liquid nitrogen into a dewar on a vacuum system of the type used for gas purification and counter filling in the chlorine experiment. The glass object in the foreground with the wire coming out that blocks Ray's left hand is an ionization gauge used to measure the pressure in the vacuum system.

involved in solar model calculations, and has always maintained a healthy skepticism regarding their validity, his interest in performing a solar neutrino experiment was the motivation for my entering and remaining in the subject. More importantly, for all of the formative years of the "solar neutrino problem", Ray inspired everyone who became involved with solar neutrinos by his conviction that valid and fundamental measurements could be made using solar neutrinos. We committed to a subject that did not attract main stream scientists because we believed in Ray's dream of measuring the solar neutrino flux.

In 1967, one year before the first results of Ray's chlorine solar neutrino experiment were announced, Bruno published a prophetic paper entitled: 'Neutrino Experiments and the Problem of Conservation of Leptonic Charge' [Zh. Exp. Teor. Fiz. 53, 1717 (1967)]. In this paper, Bruno suggested many different experiments that could test whether leptonic charge was conserved. The grandchildren of these experiments are being discussed this summer by particle physicists at conferences 


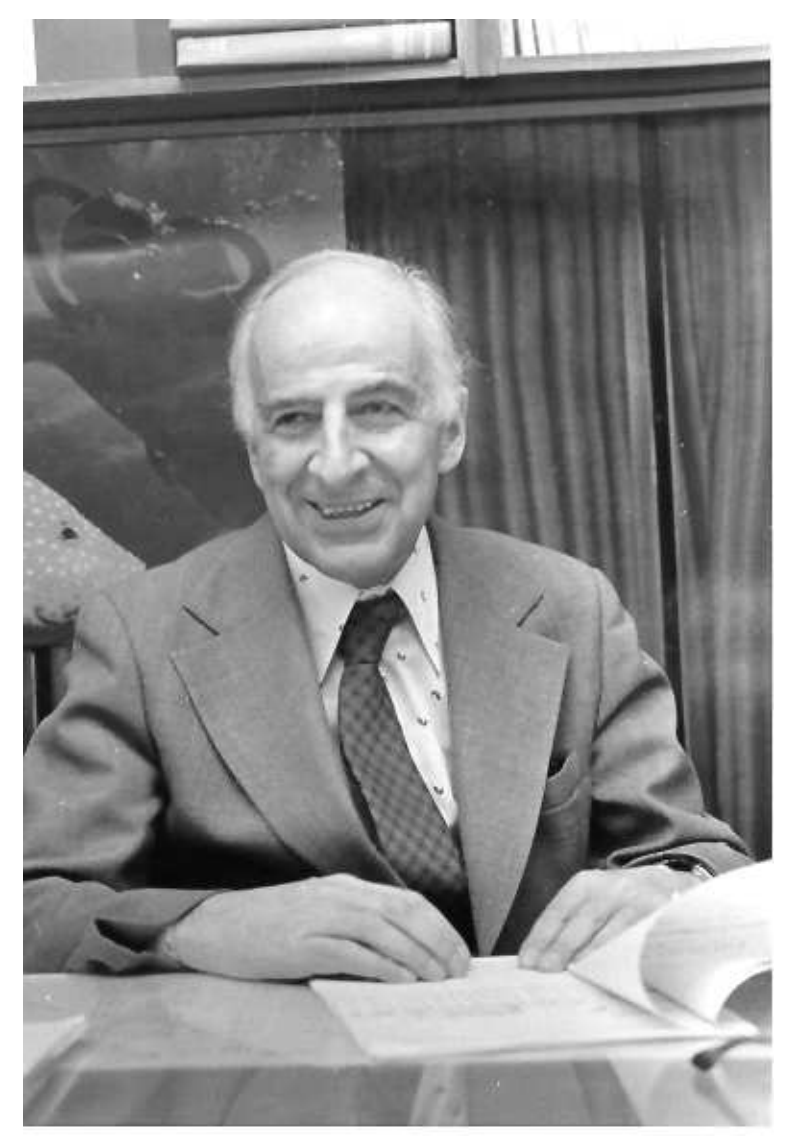

Figure 2: Bruno Pontecorvo wrote in 1967: 'From the point of view of detection possibilities, an ideal object is the sun.' Figure courtesy of S. Bilenky .

held all over the world.

Bruno included a short section in his paper that he called 'Oscillations and Astronomy.' In this section, Bruno wrote: "From the point of view of detection possibilities, an ideal object is the sun," What a wonderfully contemporary statement!

Bruno, like most particle physicists of the 1960's, the 1970's, the 1980's, and even the 1990's, did not believe astrophysical calculations could be reliable. He wrote in this same section on oscillations and astronomy: "Unfortunately, the weight of the various thermonuclear reactions in the sun, and the central temperature of the sun are insufficiently well known in order to allow a useful comparison of expected and observed solar neutrinos, from the point of view of this article." [This was 30 years before the precise confirmation of the standard solar model by helioseismology.] To support his claim, Bruno referenced only his 1946 Chalk River report, which mentioned the sun in just two sentences. Bruno did cite our detailed calculations 
of the solar neutrino fluxes elsewhere in his 1967 paper, but they seem not to have affected his thinking.

Ray and I have written three articles on the history of solar neutrino research (in 1976, 1982, and 2000, see http://www.sns.ias.edu/ jnb under the menu item Solar Neutrinos/History). It is not feasible to present in a short talk a balanced account of all the material covered in these three articles with the appropriate acknowledgments of the important work of so many people. Therefore, I shall just describe some of the highlights regarding the standard solar model from a very personal view. I encourage the listeners who are interested in a more balanced presentation to look back at the earlier articles which provide references to critical work done by a large number of researchers.

\section{The development of the "standard solar model" for neutrino predic- tions}

I describe the development of the "standard solar model" for neutrino predictions in five subsections, covering the period 1962-1988 (Section 3.1), 1988-1995 (Section 3.2), 1995-1997 (Section 3.3), 1998-2002 (Section 3.4), and 2002-2003 (Section 3.5).

\section{$3.1 \quad 1962-1988$}

At the time Ray and I first began discussing the possibility of a solar neutrino experiment, in 1962, there were no solar model calculations of solar neutrino fluxes. Ray, who heard about some of my work on weak interactions from Willy Fowler, wrote and asked if I could calculate the rate of the ${ }^{7} \mathrm{Be}$ electron capture reaction in the Sun.

After I did the calculation and submitted the paper to Physical Review, I woke up to the obvious fact that we needed a detailed model of the Sun the temperature, density, and composition profiles) in order to convert the result to a flux that Ray might consider measuring. I moved to Willy's laboratory at CalTech, where there were experts in stellar modeling who were working on stellar evolution. We used the codes of Dick Sears and Icko Iben, and a bit of nuclear fusion input that I provided, to calculate the first solar model prediction of solar neutrinos in $1962-1963$.

The result was extremely disappointing to Ray and to me, since the event rate from neutrino capture by chlorine that I calculated from our first flux evaluation was too small by an order of magnitude to be measured in any chlorine detector 
that Ray thought would be feasible. The situation was reversed in late 1963, when I realized that the capture rate for ${ }^{8} \mathrm{~B}$ neutrinos on chlorine would be increased by almost a factor of 20 over my earlier calculations because of transitions to the excited states of argon, most importantly the super-allowed transition from the ground state of ${ }^{37} \mathrm{Cl}$ to the isotopic analogue state at about $5 \mathrm{MeV}$ excitation energy in ${ }^{37} \mathrm{Ar}$. This increase in the predicted rate made the experiment appear feasible and Ray and I wrote a joint paper for Physical Review Letters proposing a practical chlorine experiment, a paper that was separated into two shorter papers to meet the space requirements.

During the period 1962 - 1968, the input data to the solar models were refined in a number of important ways as the result of the hard work of many people. The most significant changes were in the measured laboratory rate for the ${ }^{3} \mathrm{He}-{ }^{3} \mathrm{He}$ reaction (changed by a factor of 3.9), in the theoretically calculated rate for the $p-p$ reaction (changed by $7 \%$ ), and the observed value of the heavy element to hydrogen ratio, $Z / X$ (decreased by a factor of 2.5 ). Unfortunately, each of the individual corrections were in a direction that decreased the predicted flux.

Ray's first measurement was reported in PRL in 1968. Our accompanying best-estimate solar model prediction (made together with N. A. Bahcall and G. Shaviv) was about a factor of 2.5 times larger than Ray's upper limit. But the uncertainties in the model predictions were, in 1968, sufficiently large that I personally did not feel confident in concluding that the disagreement between prediction and measurement meant that something fundamental was really wrong.

As it turned out, the values of the stellar interior parameters used in 1968 are in reasonably good agreement with the values used today. However, the uncertainties are much better known now, after more than three decades of intense and precise studies and refinements by many different groups working all over the world.

The laboratory measurement of the ${ }^{7} \mathrm{Be}(p, \gamma)^{8} \mathrm{~B}$ cross section was a principal source of uncertainty in the 1962 prediction, remained a principal uncertainty in 1968, and is still today one of the two largest uncertainties in the solar neutrino predictions. Moreover, the best-estimate measured value for the cross section has decreased significantly since 1968 (see Figure 5).

As we shall see in the subsequent discussion, the only fundamentally new element that has been introduced in the theoretical calculations since 1968 is the effect of element diffusion in the sun (see 1988-1997 below).

During the period 1968 - 1988, very few people worked on topics related to solar neutrinos. There was only one solar neutrino detector, Ray's chlorine experiment. His measurement was lower than our prediction. I concentrated during 
these two long decades on refining the predictions and, most importantly, making the estimates of the uncertainties more formal and more robust.

We calculated the uncertainties by computing the partial derivatives of each of the fluxes with respect to each of the significant input parameters. In 1988, Roger Ulrich and I also did a Monte Carlo study of the uncertainties, which made use of the fluxes calculated from 1000 standard solar models. For each of the 1000 models, the value of each input parameter was drawn from a probability distribution that had the same mean and variance as was assigned to that parameter. The Monte Carlo results confirmed the conclusions reached using the partial derivatives. The uncertainty estimates made during this period are the basis for the uncertainties assigned in the current neutrino flux predictions and influence inferences regarding neutrino parameters (like $\Delta m^{2}, \tan ^{2} \theta$ ) that are derived from analyses that make use of the solar model predictions.

\section{$3.2 \quad 1988-1995$}

In the period $1990-1994, \mathrm{~F}$. Rogers and J. Iglesias of the Livermore National Laboratory published their detailed and improved calculations of stellar radiative opacities and equation of state. Now almost universally used by stellar modelers, this fundamental work resolved a number of long standing discrepancies between observations and predictions of stellar models.

In the same 1988 RMP paper in which we presented the Monte Carlo study of the uncertainties, Roger Ulrich and I also made comparisons between the predictions of our standard solar model-constructed to predict solar neutrinos-and the then existing helioseismological data on $p$-mode oscillations. The agreement was reasonably impressive: the model predictions and the measured frequency splittings agreed to about $0.5 \%$. But, we suspected that there was something missing in the solar models.

During the period 1990-1995, my colleagues and I made successively better approximations at including element diffusion in the solar model calculations. First, we derived an approximate analytic description which was included in the solar models (after some significant coding struggles) and later we made use of a precise computer subroutine that calculated the diffusion numerically. This work was done with S. Basu, A. Loeb, M. Pinsonneault, and A. Thoule. 


\section{$3.3 \quad 1995-1997$}

In 1995, Steve Tomczyk and his colleagues presented the first observations of the solar $p$-mode oscillations that included modes that sampled well both the intermediate solar interior and the deep interior. These observations determined precise observational values for the sound speed over essentially the entire solar interior.

We were in a wonderful position to make use of these precise sound speeds. In 1995, Marc Pinsonneault and I had just published a systematic study of improved solar models that incorporated the new opacity and equation of state calculations from the Livermore group and, most importantly, we had succeeded in including helium and heavy element diffusion in our standard solar model.

Together with Sarbani Basu and Joergen Christensen-Dalsgaard, we showed that the helioseismologically measured sound speeds were in excellent agreement throughout the Sun with the values calculated from our previously constructed standard solar model. As shown in Figure 3, the agreement averaged better than $0.1 \%$ r.m.s. in the solar interior. We made a simple scaling argument between accuracy in predicting sound speeds and accuracy in predicting neutrino fluxes. The concluding sentence in the Abstract of our PRL paper was:

"Standard solar models predict the structure of the Sun more accurately than is required for applications involving solar neutrinos."

This result was published in the January 1997 issue of PRL, but I had earlier presented at Neutrino '96 in Helsinki (June 1996) the same conclusion based upon somewhat less precise helioseismological data. Since some of you were present also at Helsinki, you may be interested in the precise form of the statement made in the printed proceedings:

"Helioseismology, as summarized in Figure 2 [a comparison of measured and calculated sound speeds], has effectively shown that the solar neutrino problems cannot be ascribed to errors in the temperature profile of the Sun."

The helioseismological confirmation of the standard solar model changed for me personally the way that I regarded 'the solar neutrino problem.' I no longer felt it was necessary to soften the claim that the origin of the 'problem' was new physics not bad astronomy.

So, from the astronomical perspective, we have known for six years that new physics was required to resolve the discrepancy between the standard predictions of the solar model and electroweak theory. Even prior to the existence of this helioseismological evidence, it had become clear that one could not fit the data for all the solar neutrino experiments by simply re-scaling standard predictions of 


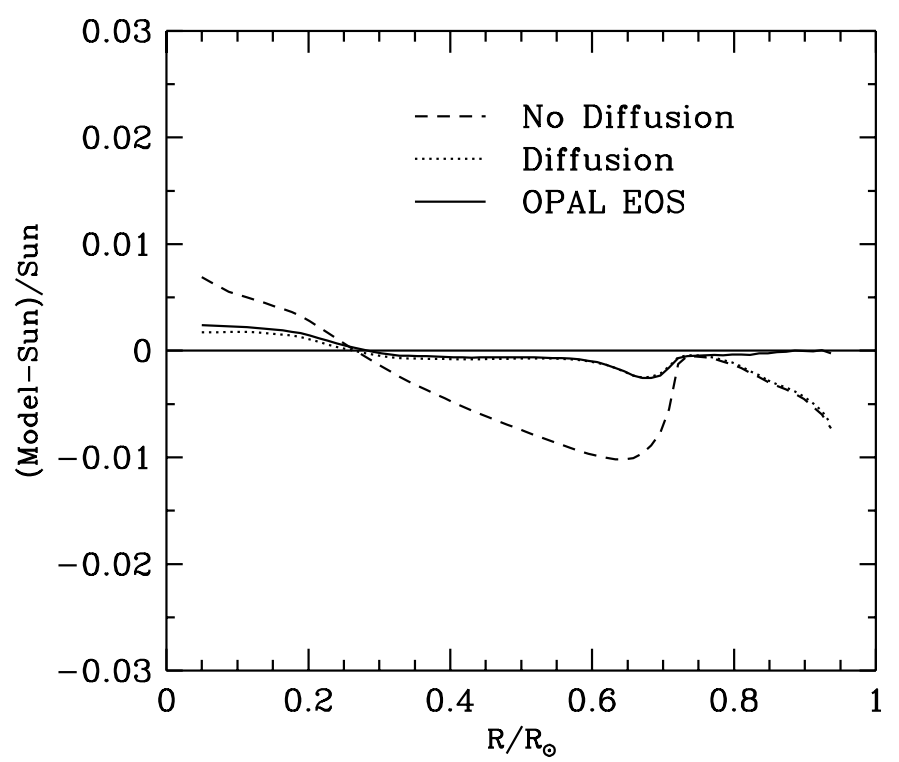

Figure 3: Comparison of measured and calculated sound speeds. This figure from astro-ph/9610250, PRL 178,171 1997 compares the sound speeds calculated with the standard solar model, BP95, with the helioseismologically determined sound speeds. The dashed curve represents the results from a solar model that does not include element diffusion. Much better agreement is obtained when element diffusion is included, as indicated by the dotted curve. The solid line represents a model in which both element diffusion and the refined OPAL equation of state are included. The inclusion of the refined equation of state results in a slight improvement in the innermost region of the solar model.

neutrino fluxes.

Why did it take so long? In Section 5, I will try to answer the question: Why were some physicists unconvinced by the astronomical evidence that solar neutrino oscillations occurred?

\section{$3.4 \quad 1998-2002$}

The SNO and Super-Kamiokande experiments have confirmed directly the calculated solar model flux of ${ }^{8} \mathrm{~B}$ neutrinos, provided there is not a large component of sterile neutrinos in the incident flux.

In units of $10^{6} \mathrm{~cm}^{-2} \mathrm{~s}^{-1}$, the standard solar model prediction for the flux, $\phi$, of rare ${ }^{8} \mathrm{~B}$ neutrinos is

$$
\phi(\mathrm{BP} 00)=5.05_{-0.8}^{+1.0}
$$

In June 2001, the SNO collaboration announced that the combined result from their 
initial CC measurement and the Super-Kamiokande $\nu-e$ scattering measurement implied a flux of ${ }^{8} \mathrm{~B}$ active neutrinos equal to

$$
\phi(\mathrm{SNO} \mathrm{CC}+\mathrm{SK})=5.44 \pm 0.99 .
$$

The agreement between the best-estimate calculated value given in Eq. (1) and the best-estimated measured value given in Eq. (2) is $0.3 \sigma$.

The recent SNO NC measurement implies an even closer agreement between the best-estimates. Assuming an undistorted ${ }^{8} \mathrm{~B}$ neutrino spectrum (a very good approximation), the SNO collaboration finds

$$
\phi(\mathrm{NC})=5.09 \pm 0.64 .
$$

The agreement between the best-estimates given in Eq. (1) and Eq. (3) is embarrassingly small, $0.03 \sigma$, but obviously accidental. The quoted errors, theoretical and experimental, are real and relatively large.

\subsection{2-2003:Post-KamLAND}

Very recently, the results of the KamLAND reactor experiment (hep-ex/0212021) have led to a more precise determination of both the ${ }^{8} \mathrm{~B}$ and (after this meeting was held) the $p-p$ neutrino fluxes.

We have recently completed a global analysis of all the available solar and reactor data (see hep-ph/0212147 and hep-ph/0305159), including especially the KamLAND measurements. The agreement with the standard solar model predictions is good. When expressed in terms of the standard solar model (BP00) predicted neutrino flux, the experimentally determined flux of ${ }^{8} \mathrm{~B}$ solar neutrinos is

$$
\phi\left({ }^{8} \mathrm{~B}\right)=1.00 \pm 0.04
$$

The experimentally-determined flux of $p-p$ solar neutrinos, expressed in terms of the BP00 predicted flux, is

$$
\phi(\mathrm{p}-\mathrm{p})=1.01 \pm 0.02
$$

We shall now discuss uncertainties in the predictions of the solar neutrino fluxes. 


\section{Uncertainties in the solar model predictions}

I will begin the discussion of uncertainties with a brief introduction in Section 4.1 that emphasizes the importance of robust and well-defined estimates of the errors. Then I will describe in Section 4.2 the most important sources of uncertainties in the contemporary predictions.

\subsection{Skepticism}

From the very beginning of solar neutrino research, the uncertainties in the solar model predictions have been a central issue. We could not learn things about the Sun or about neutrinos using solar neutrino experiments unless we could demonstrate that the uncertainties in the predictions were small and robustly calculated. If, as many physicists initially believed, the astronomical predictions were not quantitatively reliable, then there was no real "solar neutrino problem."

Bruno Pontecorvo, in his prophetic paper "Neutrino Experiments and the Problem of Conservation of Lepton Charge", Soviet Physics JETP, 26, 984 (1968), expressed the view that the uncertainties in the solar model calculations were so large as to prevent a useful comparison with solar neutrino experiments, Here is what Bruno said:

"From the point of view of detection possibilities, an ideal object is the sun... Unfortunately, the weight of the various thermonuclear reactions in the sun, and the central temperature of the sun, are insufficiently well known in order to allow a useful comparison of expected and observed solar neutrinos, from the point of view of this article."

This comment by Bruno Pontecorvo is indicative of the skepticism about solar model predictions that existed among many physicists. In an effort to remove this skepticism, I spent much of the 34 years from 1968 to 2002 refining the predictions of the solar neutrino fluxes and providing increasingly more robust estimates of the uncertainties in the predictions.

I want to summarize for you now the current best estimates for the uncertainties in the solar neutrino predictions.

\subsection{Currently estimated uncertainties in predicted neutrino fluxes}

I will first present in Section 4.2.1 the current values for the total and the partial uncertainties in the flux predictions. Then I will describe in Section 4.2.2 and Section 4.2.3, respectively, the very different histories for the determination of the 


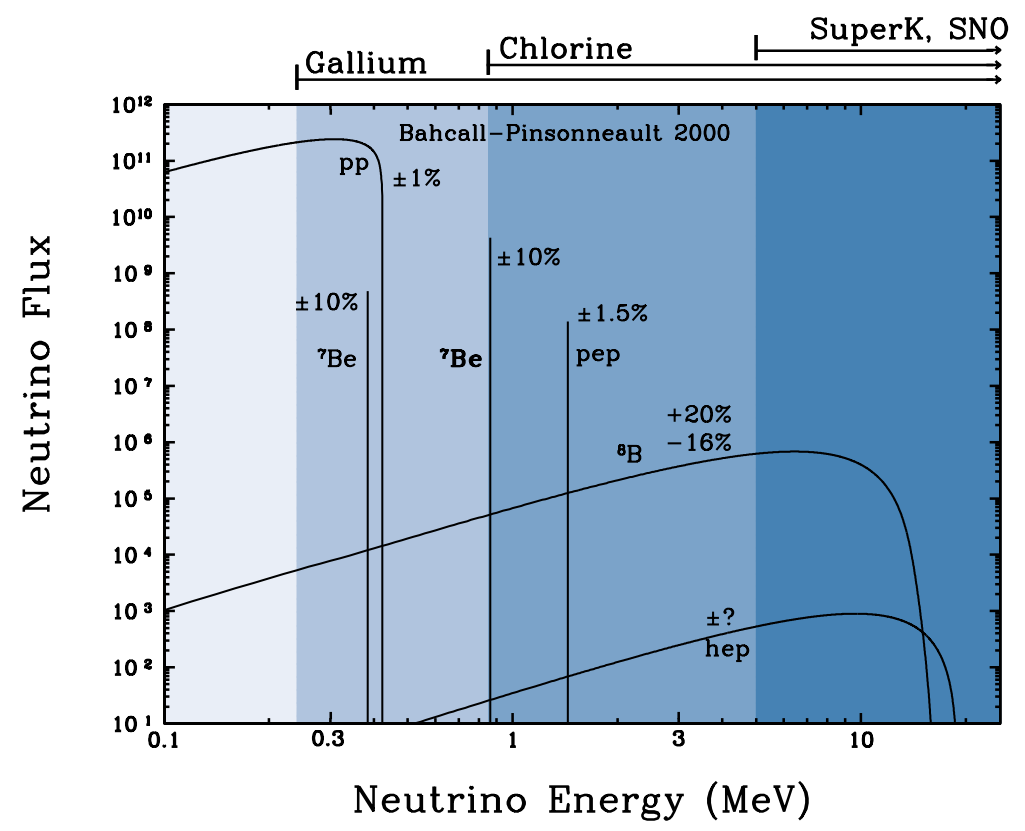

Figure 4: Solar neutrino spectrum with currently estimated uncertainties.

cross sections for the ${ }^{7} \mathrm{Be}(\mathrm{p}, \gamma){ }^{8} \mathrm{~B}$ reaction and the ${ }^{37} \mathrm{Cl}\left(\nu, e^{-}\right)^{37} \mathrm{Ar}$.

\subsubsection{Total and fractional uncertainties}

Figure 4 shows the calculated values for the principal (p-p) solar neutrino fluxes and their estimated uncertainties. The $\mathrm{p}-\mathrm{p}$ and pep neutrino fluxes are predicted with a calculated uncertainty of only $\pm 1 \%$ and $\pm 1.5 \%$, respectively. The ${ }^{7}$ Be neutrino flux is predicted with an uncertainty of $\pm 10 \%$ and the important ${ }^{8} \mathrm{~B}$ neutrino flux, which is measured by Super-Kamiokande and SNO, is predicted with an error of about $20 \%$. The fluxes from CNO reactions, especially ${ }^{13} \mathrm{~N}$ and ${ }^{15} \mathrm{O}$ neutrino fluxes, are predicted with less precision than the fluxes from the p-p reactions. I have not shown the CNO fluxes in Figure 4 since these fluxes are not expected to play a discernible role in any of the planned or in progress solar neutrino experiments.

Table 1 shows how much each of the principal sources of uncertainty contribute to the total present-day uncertainty in the calculation of the ${ }^{8} \mathrm{~B}$ and ${ }^{7} \mathrm{Be}$ solar neutrino fluxes. The largest uncertainty in the prediction of the ${ }^{8} \mathrm{~B}$ neutrino flux is caused by the estimated error in the laboratory measurement of the low energy cross section for the ${ }^{7} \mathrm{Be}(\mathrm{p}, \gamma){ }^{8} \mathrm{~B}$ reaction (This statement was also true in 1962 , $1964,1968, \ldots$.$) . The largest uncertainty in the prediction of the { }^{7}$ Be neutrino flux is due to the quoted error in the measurement of the low energy rate for the ${ }^{3} \mathrm{He}+$ 
Table 1: Fractional uncertainties in the Predicted ${ }^{8} \mathrm{~B}$ and ${ }^{7}$ Be Solar Neutrino Fluxes (BP00). The table presents the fractional uncertainties in the calculated ${ }^{8} \mathrm{~B}$ and ${ }^{7}$ Be neutrino fluxes, due to the different factors listed in the column labeled Source. The first four rows refer to the low energy cross section factors for different fusion reactions. The last four rows refer to the heavy element to hydrogen ratio, $Z / X$ (Composition), the radiative opacity, a multiplicative constant in the expression for the diffusion rate of heavy elements and helium, and the total solar optical luminosity.

\begin{tabular}{ccc}
\hline \hline Source & ${ }^{8} \mathrm{~B}$ & ${ }^{7} \mathrm{Be}$ \\
\hline $\mathrm{p}-\mathrm{p}$ & 0.04 & 0.02 \\
${ }^{3} \mathrm{He}+{ }^{3} \mathrm{He}$ & 0.02 & 0.02 \\
${ }^{3} \mathrm{He}+{ }^{4} \mathrm{He}$ & 0.08 & 0.08 \\
$\mathrm{p}+{ }^{7} \mathrm{Be}$ & ${ }_{-0.07}^{+0.14}$ & 0.00 \\
Composition & 0.08 & 0.03 \\
Opacity & 0.05 & 0.03 \\
Diffusion & 0.04 & 0.02 \\
Luminosity & 0.03 & 0.01 \\
\hline \hline
\end{tabular}

${ }^{4}$ He reaction. In addition, there are a number of other sources of uncertainty, all of which contribute more or less comparably to the total uncertainty in the prediction of the ${ }^{7} \mathrm{Be}$ and the ${ }^{8} \mathrm{~B}$ neutrino fluxes.

\subsubsection{The saga of the ${ }^{7} \mathrm{Be}(p, \gamma)^{8} \mathrm{~B}$ cross section}

Figure 5 shows, as a function of the date of publication, measured values for the low energy cross section of the crucial reaction ${ }^{7} \mathrm{Be}(\mathrm{p}, \gamma){ }^{8} \mathrm{~B}$. [Some very recent measurements are included in the reference nucl-ex/0212011.] I have only shown here the direct measurements of this reaction; there are also indirect measurements that yield similar results.

The encouraging aspect of Figure 5 is that the huge uncertainty that existed between 1960 and 1980, of order a factor of two, has been much reduced in the following two decades. In the BP00 calculations, we adopted as the best-estimate the Adelberger et al. [RMP, 70, 1265 (1998), astro-ph/9805121] consensus value for the cross section factor of the ${ }^{7} \mathrm{Be}(\mathrm{p}, \gamma)^{8} \mathrm{~B}$ reaction, $S_{17}(0)=19\left(1+{ }_{-0.07}^{+0.14}\right) \mathrm{eV}-\mathrm{b}$ (the $1 \sigma$ error given here is one-third the Adelberger et al. $3 \sigma$ estimate). This value is indicated in the figure by arrow next to "Standard."

Several refined experiments are in progress or are planned to measure more accurately the low energy cross section factor for the ${ }^{7} \mathrm{Be}(\mathrm{p}, \gamma)^{8} \mathrm{~B}$ reaction or the 


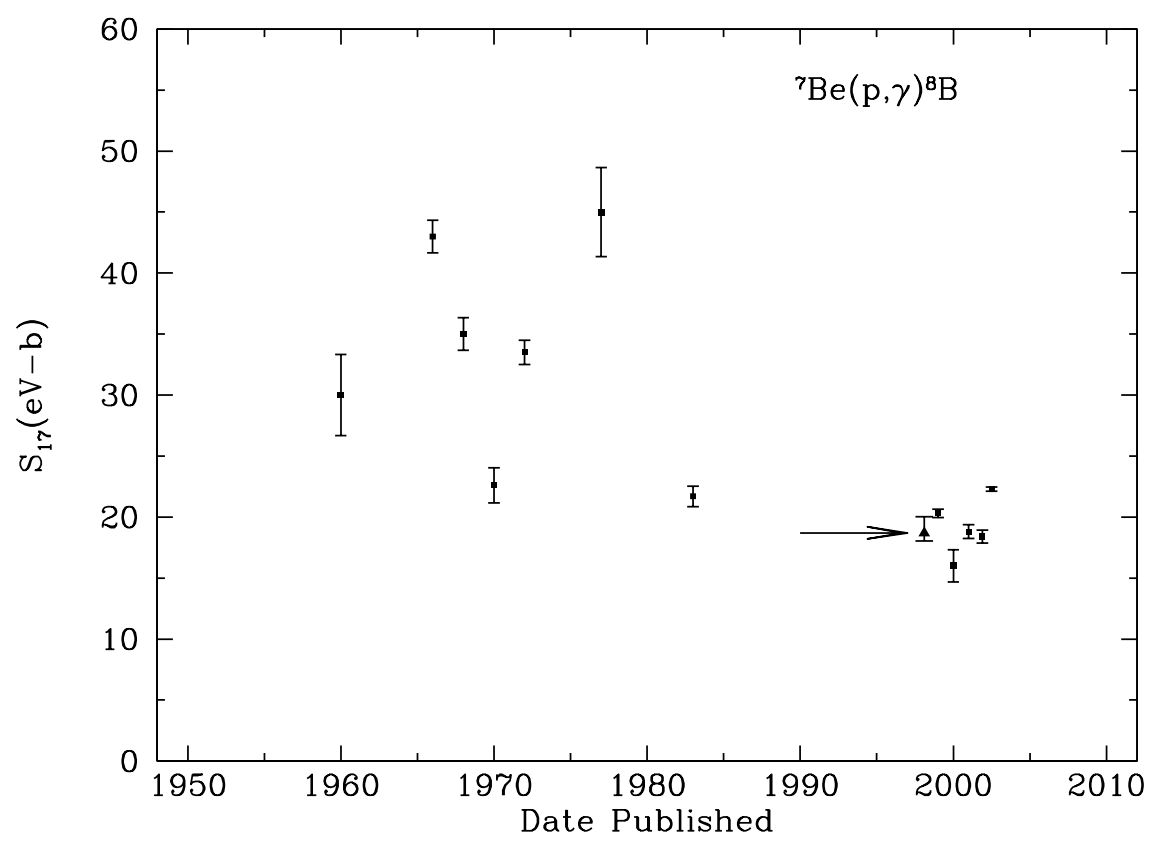

Figure 5: ${ }^{7} \mathrm{Be}(\mathrm{p}, \gamma){ }^{8} \mathrm{~B}$. The figure shows the measured values as a function of date of publication for the low-energy cross section factor for the ${ }^{7} \mathrm{Be}(\mathrm{p}, \gamma){ }^{8} \mathrm{~B}$ reaction. The arrow points to the currently standard value, recommended by Adelberger et al., that is used in the BP00 calculations. Recent precision measurements yield values slightly larger than the value recommended by Adelberger et al. but are consistent with the earlier estimate [see Phys. Rev. Lett. 90 (2003) 022501 and Table 2 of nucl-ex/0212011.]

$\mathrm{p}\left({ }^{7} \mathrm{Be}, \gamma\right)^{8} \mathrm{~B}$ reaction. Also, there are a number of related reactions that are being studied in order to give somewhat more indirect information about the low energy cross section. The goal of all these experiments is to reduce the combined systematic and statistical errors to below $5 \%$, so that $S_{17}(0)$ is no longer a dominant source of uncertainty in the prediction of the ${ }^{8} \mathrm{~B}$ solar neutrino flux (cf. Table 1 above).

To the best of my knowledge, the preliminary data from all of the existing experiments are consistent with the currently standard value of $S_{17}(0)$ quoted above and with the best-estimate recommended in reference nucl-ex/0212011. In order to avoid the confusion that would be created by introducing numbers in the literature that are changed frequently, I prefer not to revise the "standard" estimate of $S_{17}(0)$ (and the ${ }^{8} \mathrm{~B}$ solar neutrino flux) until the in-progress experiments on ${ }^{7} \mathrm{Be}(\mathrm{p}, \gamma)^{8} \mathrm{~B}$ and related reactions are completed. 


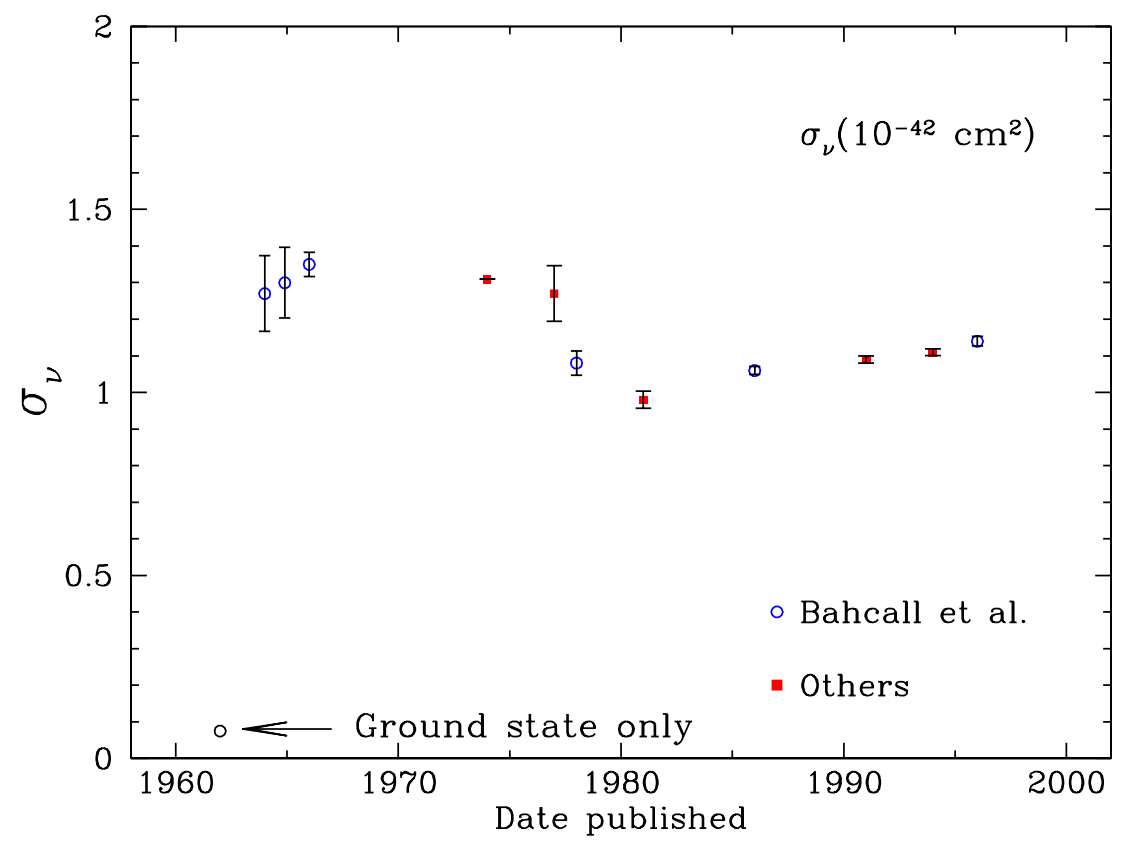

Figure 6: ${ }^{37} \mathrm{Cl}\left(\nu_{e}, e^{-}\right){ }^{37} \mathrm{Ar}$. The figure shows, for an undistorted ${ }^{8} \mathrm{~B}$ solar neutrino spectrum, the calculated values for the cross section ${ }^{37} \mathrm{Cl}\left(\nu_{e}, e^{-}\right)^{37} \mathrm{Ar}$ as a function of date of publication.

\subsubsection{The ${ }^{37} \mathrm{Cl}\left(\nu_{e}, e^{-}\right)^{37}$ Ar cross section}

In the early days of solar neutrino astronomy, the cross sections for neutrino absorption by chlorine, ${ }^{37} \mathrm{Cl}\left(\nu_{e}, e^{-}\right){ }^{37} \mathrm{Ar}$, were an important source of uncertainty. For comparison with Figure 5, I show in Figure 6 the calculated values of the absorption cross section for ${ }^{8} \mathrm{~B}$ neutrinos incident on ${ }^{37} \mathrm{Cl}$. The first calculation I made (in 1962) was too small, because I did not consider transitions to excited states. The calculation I made in 1964 was quickly confirmed by measurements made (by Poskanzer et al.) on the predicted decay: ${ }^{37} \mathrm{Ca} \rightarrow{ }^{37} \mathrm{~K}+e^{+}+\nu_{e}$, which is the isotopic analogue of the neutrino capture reaction. A series of subsequent refined measurements and calculations reduced the estimated error in the neutrino cross section to where it is no longer one of the largest sources of uncertainty in the calculation of the predicted capture rate in the chlorine solar neutrino experiment (although the uncertainty still plays some role in the global determination of solar neutrino oscillation parameters). 
4.3 Using p-p and ${ }^{7}$ Be neutrinos to probe details of solar fusion

Is there any way of probing the solar interior and determining experimentally which terminating reaction of the p-p chain, ${ }^{3} \mathrm{He}-{ }^{3} \mathrm{He}$ or ${ }^{3} \mathrm{He}-{ }^{4} \mathrm{He}$, is faster in the solar interior and by how much? Yes, there is a way. Solar neutrino experiments can do just that.

The ratio $\mathrm{R}$ of the rate of ${ }^{3} \mathrm{He}-{ }^{3} \mathrm{He}$ reactions to the rate of ${ }^{3} \mathrm{He}-{ }^{4} \mathrm{He}$ reactions averaged over the Sun can be expressed in terms of the p-p and ${ }^{7}$ Be neutrino fluxes by the following simple relation ${ }^{2}$ :

$$
R \equiv \frac{<^{3} \mathrm{He}+{ }^{4} \mathrm{He}>}{<^{3} \mathrm{He}+{ }^{3} \mathrm{He}>}=\frac{2 \phi\left({ }^{7} \mathrm{Be}\right)}{\phi(\mathrm{pp})-\phi\left({ }^{7} \mathrm{Be}\right)} .
$$

The standard solar model predicts $R=0.174$. One of the reasons why it is so important to measure accurately the total p-p and ${ }^{7} \mathrm{Be}$ neutrino flux is in order to test this detailed prediction of standard solar models. The value of $\mathrm{R}$ reflects the competition between the two primary ways of terminating the p-p chain and hence is a critical probe of solar fusion.

\section{$5 \quad$ Why did it take so long?}

In the introduction to this talk, I said that I would address the question of why it took so long, about 35 years, to convince many physicists that solar neutrino research was revealing something new about neutrinos. I will now do my best to explain why the process from discovery to consensus required more than three decades.

In the early years, after the very rapid progress between 1964 to 1968, there were many things that had to be studied very carefully to see if there could be something important that had been left out of the standard solar models. The values of all of the (large number of) important input parameters were remeasured or recalculated more accurately, a variety of imaginative "non-standard solar models" were examined critically, and possible instabilities in the solar interior were investigated. It took about 20 years, 1968-1988, for the collective efforts of many nuclear physicists, atomic physicists, astronomers, and astrophysicists to provide a thoroughly explored basis for the standard model calculations that allowed robust estimates of the uncertainties in the solar model predictions. Even after this long struggle with details was mostly complete, it was still necessary to develop codes that could include the refinement of element diffusion (which took until 1995). And,

\footnotetext{
${ }^{2}$ More precisely, $\phi\left({ }^{7} \mathrm{Be}\right)$ should be replaced by the sum of the ${ }^{7} \mathrm{Be}$ and ${ }^{8} \mathrm{~B}$ neutrino fluxes in the denominator of Eq. (6).
} 
presumably, there are still today even further refinements that are appropriate and necessary to make to obtain a still more accurate description of the region in which solar fusion takes place.

My impression is that nearly all particle physicists remained blissfully unaware of, or indifferent to, the decades of efforts to make the solar neutrino predictions more robust. Why? Why did many (but not all) particle physicists not take the "solar neutrino problem" seriously?

I think that there were three reasons it took so long for particle physicists to acknowledge that new physics was being revealed in solar neutrino research. First, the Sun is an unfamiliar accelerator. Particle physicists, and most other physicists too, were skeptical of what astronomers and astrophysicists could learn about an environment that they could neither visit nor manipulate. These physicists often had only a newspaper-level understanding of the observational phenomena that stellar models reproduced and the constraints they met. Second, physicists who heard talks on solar neutrinos, were most impressed by the fact that the ${ }^{8} \mathrm{~B}$ solar neutrino flux depended on the 25 th power of the central temperature, $\phi\left({ }^{8} \mathrm{~B}\right) \propto T^{25}$. This dependence seemed to many physicists too sensitive to allow an accurate prediction (an objection which was answered experimentally only by the helioseismological measurements in 1995 and their successful comparison in 1996-1997 with standard solar model predictions, see Section 3.3.) Third, the simplest interpretation of the discrepancy between observed and predicted solar neutrino event rates, vacuum neutrino oscillations (proposed by Bruno Pontecorvo), suggested large mixing angles for the neutrinos. It was widely (but not universally) agreed among particle theorists that mixing angles in the lepton sector would be small in analogy with the mixing angles in the quark sector. The most popular view of particle theorists over most of the history of solar neutrino research has been that since quarks and leptons are probably in the same multiplets, they should have mixing angles of comparable size. This objection to new solar neutrino physics was removed only when Mikheyev and Smirnov built upon the earlier work of Wolfenstein to describe the magic of the MSW effect. Ironically, the small mixing angle (SMA) MSW solution persuaded a significant number of physicists that there might be new physics being revealed by solar neutrino experiments, although today we know that only large mixing angles solutions are good fits to all the available solar and reactor neutrino data.

I think that the spirit with which many particle physicists regarded solar neutrino research is best expressed by a quotation from the introduction of a 1990 paper written by H. Georgi and M. Luke [Nucl. Phys. B, 347, 1 (1990)]. They began their article as follows: 
"Most likely, the solar neutrino problem has nothing to do with particle physics. It is a great triumph that astrophysicists are able to predict the number of ${ }^{8} \mathrm{~B}$ neutrinos to within a factor of 2 or $3 \ldots .$.

This writeup is based on my talks at PIC03, at Neutrino2002 (Munich) and at Venice Telescopes 2003 (organized by Milla Baldo Ceolin). This work was partially supported by an NSF grant No. PHY0070928. 